\title{
Plantes ichtyotoxiques et particularisme des usages au Congo (Brazzaville)
}

\author{
Victor KIMPOUNI ${ }^{*}$, Ernest APANI et Marcel MOTOM \\ École normale supérieure, Université Marien Ngouabi, B. P. 237, Brazzaville, Congo. \\ *Auteur Correspondant, E-mail : vkimpouni@yahoo.com
}

\section{RESUME}

L'étude ethno-anthropologique de l'usage de la biodiversité végétale, notamment des plantes ichtyotoxiques, chez 18 communautés ethnico-linguistiques de 7 groupes ethniques forestiers et savanicoles, met en évidence 26 taxons appartenant à 11 familles. Elle révèle en outre, pour la première fois dans la contrée, les propriétés ichtyocides des espèces de savane et dans une moindre proportion certaines forestières. Excepté Euphorbia trigona et Tephrosia vogelii qui sont cultivées et utilisées concomitamment par les communautés forestières et savanicoles, toutes les plantes sont spontanées. Exploitant les plantes à portée de la main, on observe des spécificités liées à l'habitat. Au regard du mode d'utilisation, chacune d'elles est une recette à part entière. Ainsi nous avons 6 (soit 23,07\%) recettes spécifiques en savane, contre 17 (soit 65,38\%) en forêt. Les données ethnobotaniques montrent qu'une dizaine de taxons, dont les vertus piscicides sont prouvées ailleurs, existent dans la contrée sans faire l'objet d'une exploitation. Les communautés forestières et savanicoles exploitent activement la flore, pour assurer leur survie. L'usage de la plupart de ces ichtyocides requiert quelques précautions qui sans être observées engendrent des intoxications, parfois violentes à forte dose, suite à la consommation du poisson.

() 2011 International Formulae Group. All rights reserved.

Mots clés : Congo, ethno-anthropologie, biodiversité végétale, ichtyocide, intoxication, recette.

\section{INTRODUCTION}

Le Congo couvre $342.000 \mathrm{~km}^{2}$ en Afrique centrale et s'étend de part et d'autre de l'équateur du sud-ouest $\left(11^{\circ} 11^{\prime} \mathrm{E}, 5^{\circ} 00^{\prime}\right.$ S) au nord-est $\left(18^{\circ} 35^{\prime}\right.$ E, $\left.3^{\circ} 34^{\prime} \mathrm{N}\right)$. Situé en plein cœur des forêts du bassin du Congo, sa couverture végétale est formée de 60 à $65 \%$ de forêts et 35 à $40 \%$ de savanes (Vennetier, 1977 ; UICN, 1996). Le Congo est limité au nord par le Cameroun et la Centrafrique, à l'est et au sud par le Congo Démocratique, au sud-ouest par l'enclave du Cabinda et la façade maritime de l'océan atlantique (170 $\mathrm{km}$ ) et enfin à l'ouest par le Gabon (Figure 1). Le territoire congolais est parcouru par un réseau hydrographique très important aux cours d'eau et lacs poissonneux. La pêche continentale se pratique de manière presque artisanale et recourt aux instruments comme les filets à mailles variables, les hameçons, les nasses, etc. Outre les techniques de pêche usant de ces outils qui sont presque exclusivement la panacée des hommes en général; les femmes quant à elles, usent le plus souvent des techniques empiriques allant de l'écope à l'usage des plantes ichtyotoxiques.

Loin d'être des zones inhabitées, les forêts et les savanes hébergent des populations autochtones et locales qui profitent des bioressources les constituant. Elles y trouvent 
une source de protéines, de biens et de services d'usages quotidiens. Les enquêtes ethnobotaniques auprès des différentes populations vivant dans ces milieux ont permis de mettre en évidence les aspects anthropologiques tenant à l'usage des plantes sauvages (Grenand et Prévost, 1994). Parmi ces recherches relatives à l'homme et son environnement, nous relevons l'alimentaire, la phytothérapie, le culturel, l'artisanal, les poisons de chasse et de pêche (Kimpouni et Nguembo, sous presse).

La revue de la littérature montre que tous ces aspects ne font pas l'objet d'un traitement égal. Comme le témoigne la disponibilité en quantité et qualité de la documentation, les efforts investis sont en faveur des plantes médicinales et alimentaires. Outre ces deux aspects, les autres volets des recherches ethnobotaniques semblent être considérés comme marginaux (Grenand et Prévost, 1994). En vue de cerner les savoirs (être, faire et vivre) de ces populations, nous soulevons dans ce travail, le volet des plantes ichtyotoxiques qui est plus que discret lors des enquêtes ethnobotaniques (Grenand et Prévost, 1994). En effet, celles-ci mettent en valeur les connaissances endogènes des populations autochtones et locales, sur la gestion et l'exploitation de la biodiversité végétale.

En Afrique centrale et généralement dans le Bassin du Congo, les données sur les plantes ichtyotoxiques sont très globalisantes. Lorsque des spécificités sont référenciées, rien ou presque n'est dit sur le Congo. Les données existantes quoique disparates et fragmentaires, intéressent principalement les populations forestières, comme si celles des savanes n'exploitent pas les vertus de la diversité floristique dans la satisfaction quotidienne de leurs besoins. C'est ainsi que cette étude vient combler le hiatus longtemps entretenu sur les connaissances et les savoirs endogènes des communautés forestières et savanicoles du territoire congolais. La maîtrise des données anthropologiques rime avec l'authentification de l'identité socioculturelle des communautés locales et autochtones, synonyme des acquis sur l'exploitation de la diversité floristique (Grenand et Prévost, 1994).

\section{Objectif de l'étude}

Cette étude vise comme objectif, la capitalisation des savoirs endogènes des communautés et des groupes ethnicolinguistiques du Congo. Elle se base sur l'exploitation des valeurs socioculturelles liées à la biodiversité végétale.

\section{MATERIEL ET METHODES \\ Matériel}

Le matériel d'étude est constitué des plantes citées et retenues comme ichtyotoxiques par les communautés ou par des personnes âgées adeptes de ces pratiques de pêche. Ce matériel bien connu des populations est récolté, identifié systématiquement (Planche 1) et ordonné suivant l'APG III (Angiosperm phylogeny group, 2009). Les éléments notés pour chaque espèce sont: la partie utilisée, le mode opératoire et le nom vernaculaire.

\section{Méthodologie}

L'enquête ethnobotanique se focalise sur la connaissance que les membres des groupes ethniques/linguistiques du Congo, riverains des cours d'eau expriment sur les vertus de la flore avoisinante, notamment les ichtyocides. Le panel d'informateurs se base sur la mixité de genres et la valeur empirique intrinsèque de chacun d'eux sur la connaissance des végétaux et de leurs vertus. Son socle se compose principalement d'adultes et des personnes âgées ayant une expérience dans l'usage des produits ichtyotoxiques. Le nombre d'informateurs, très limité, n'est pas fonction de l'importance de la localité en termes de résidents, mais plutôt en nombre d'individus possesseurs des secrets des plantes.

Les enquêtes portent sur les interviews personnalisées et les «focus group » impliquant les deux genres. Elles sont semi directives et à questions ouvertes, sans influence sur la réponse attendue de l'enquêté (Martin, 1995). Au cours de cet exercice, la démarche ethnobotanique consiste à identifier la ressource et à connaître les propriétés qui sont associées ou qui ont été associées. Enfin, elle est ponctuée par les opérations de collecte des spécimens de la ressource et le suivi des 
différents modes de traitement et d'usage du produit. Cette procédure permet de sérier et d'authentifier l'information reçue et si possible, de la matérialiser en relevant les signes tangibles d'exploitation. Au cours de cet exercice, nous notons les parties de la plante utilisées.

Afin de suivre l'information et de la recouper, nous notons le groupe ethnique et l'origine de nos informateurs. Selon la répartition de Bouquet (1969), cette étude intéresse 18 communautés ethnicolinguistiques représentant 7 groupes ethniques du Congo, disséminés à travers toutes les zones forestières et savanicoles. Il s'agit du :

Groupe ethnique Koongo avec 10 communautés ethnico-linguistiques (1: Mvili, 2: Yombe, 3: Kouni, 4: Kaamba, 5: Doondo, 6: Beembe, 7 : Minkengue, 8 : Suundi, 9 : Hangala, 10 : Koongo), dont seul le Yombé vit en forêt;

- Groupe ethnique Téké (20: Yaka, 21: Laali) ;

- Groupe ethnique Mbôsi (33 : Mbochi, 37 :

Mboko);

- Groupe ethnique Sangha (46 : Enyelle);

- Groupe ethnique Mbéré (18: Ngaré);

- Groupe ethnique Makaa (64 : Bakouele);

- Groupe ethnique Punu (14 : Loumbou).

\section{RESULTATS}

Répartition taxinomique et écologique

L'inventaire relève 26 espèces angiospermiennes pour 11 familles (Tableau 1). Suivant la répartition des espèces par habitat, on compte 9 espèces forestières (60\%), 4 de savanes (27\%) et 2 cultivées (13\%). L'exploitation des vertus ichtyotoxiques montre que l'usage des espèces forestières est une pratique courante des populations riveraines, tant de la partie septentrionale que du sud-ouest. Excepté Tephrosia vogelii dont la valeur est commune à toutes les communautés, l'usage des espèces savanicoles et ripicoles est une pratique presque exclusive des populations du sud.

\section{Diversité floristique}

L'analyse de la diversité floristique met en relief 3 familles (Arecaceae,
Euphorbiaceae et Fabaceae) fournissant 59\% d'espèces ichtyotoxiques (Figure 2). Les moins pourvues ont à peine $15 \%$ du total de l'inventaire et ce sont: Acanthaceae, Asparagaceae, Costaceae, Iridaceae et les Rubiaceae.

\section{Importance des organes}

Les organes utilisés sont très diversifiés et les fruits sont les plus exploités (Figure 3). $63 \%$ des espèces piscicides inventoriées par les fruits et les tiges, $25 \%$ par les feuilles et les écorces. Les autres parties réunies n'intervenant que pour $11 \%$.

\section{Distribution des types morphologiques}

La distribution des espèces en types morphologiques montre que les ligneux fournissent le grand lot de matériel (Figure 4). L'importance des types morphologiques varie de 13,33 à 46,67\%, soit de 2 à 4 espèces. Les arbres et les arbustes pris ensemble englobent environ 73,33\% (soit 11 espèces), alors que les herbes vivaces sont les moins communes.

\section{Mode et forme d'usage}

L'analyse des modes et formes d'usage met en évidence deux groupes de plantes. L'organe est utilisé naturel, sans la moindre transformation. Dans ce cas, on relève la tige d'Euphorbia trigona qui est coupée directement en petits morceaux afin de répandre le latex dans l'eau. Quant aux autres espèces, Euphorbia trigona y comprise, fournissent un produit frais, rendu en pâte par pilage des tiges, des écorces, des fruits, des feuilles et des bulbes. Le produit final peut subir suivant les cas une maturation à température ambiante, à l'abri de la lumière et du soleil. Cette phase de maturation dure 1 à 2 jours, avant usage. Cette observation qui consiste à fermenter le produit, suivant un certain délai, est répertoriée chez plusieurs communautés. Quant aux produits des Raphia $s p$., les noix encore vertes sont simplement fermentées à température ambiante et à l'abri de la lumière, avant la mise à l'eau. Soulignons que ce produit est très toxique, même pour l'homme. 
Tableau 1 : Caractères bioécologiques et ethnologiques des plantes Ichtyotoxiques en usage.

\begin{tabular}{|c|c|c|c|c|c|c|}
\hline Taxons et diversité spécifique & $\begin{array}{l}\text { Habitat } \\
\text { \& statut }\end{array}$ & P.U. & T.M. & T.P. & $\begin{array}{l}\text { Noms } \\
\text { vernaculaires }\end{array}$ & $\begin{array}{l}\text { Aire } \\
\text { d'usage }\end{array}$ \\
\hline \multicolumn{7}{|l|}{ Acanthaceae (1/4\%) } \\
\hline Justicia extensa T.Anders & Culture & Feuille & ar & Aftr & $\begin{array}{l}\text { Mbaka, } \\
\text { Lengeti, } \\
\text { Lembaha }\end{array}$ & Cuvette \\
\hline \multicolumn{7}{|l|}{ Arecaceae (6 / 24\%) } \\
\hline $\begin{array}{l}\text { Calamus deeratus Mann \& } \\
\text { Wendl.* }\end{array}$ & Forêt & $\begin{array}{l}\text { Tige } \\
\text { fraîche }\end{array}$ & $\mathrm{L}$ & $\mathrm{GC}$ & Mbâmba & Pool \\
\hline Eremospatha cabrae De Wild.* & Forêt & $\begin{array}{l}\text { Tige } \\
\text { fraîche }\end{array}$ & $\mathrm{L}$ & $\mathrm{CG}$ & Mbâmba & Pool \\
\hline $\begin{array}{l}\text { Eremospatha wendlandiana } \\
\text { Dammer ex Becc.* }\end{array}$ & Forêt & $\begin{array}{l}\text { Tige } \\
\text { fraîche }\end{array}$ & $\mathrm{L}$ & $\mathrm{GC}$ & Mbâmba & Pool \\
\hline $\begin{array}{l}\text { Eremospatha hookeri (Mann \& } \\
\text { Wendl.) Wendl.* }\end{array}$ & Forêt & $\begin{array}{l}\text { Tige } \\
\text { fraîche }\end{array}$ & $\mathrm{L}$ & $\mathrm{GC}$ & Mbâmba & Pool \\
\hline Raphia sp. & $\begin{array}{l}\text { Forêt } \\
\text { inondée }\end{array}$ & $\begin{array}{l}\text { Fruit } \\
\text { vert }\end{array}$ & ar & & (Tiam-tiam) & Cuvette \\
\hline Raphia vinifera* & $\begin{array}{l}\text { Forêt } \\
\text { inondée }\end{array}$ & $\begin{array}{l}\text { Fruit } \\
\text { vert }\end{array}$ & ar & & $\begin{array}{l}\begin{array}{l}\text { Kolo } \\
\text { (Molengé) }\end{array} \\
\end{array}$ & Cuvette \\
\hline \multicolumn{7}{|l|}{ Iridaceae $(1 / 4 \%)$} \\
\hline $\begin{array}{l}\text { Gladiolus gregarius Welw. ex } \\
\text { Baker }\end{array}$ & Savane & $\begin{array}{l}\text { Rhizome } \\
\text { (bulbe) }\end{array}$ & $\mathrm{Hv}$ & Aftr & $\begin{array}{l}\text { Kiluanga, } \\
\text { Boumi, } \\
\text { Luanga-luanga }\end{array}$ & $\begin{array}{l}\text { Bouenza } \\
\text { Niari }\end{array}$ \\
\hline \multicolumn{7}{|l|}{ Asparagaceae (1 / 4\%) } \\
\hline Urginea altissima (L.f.) Bak. & Savane & Bulbe & $\mathrm{Hv}$ & $\mathrm{Cg}$ & Mbaka & $\begin{array}{l}\text { Bouenza, } \\
\text { Niari }\end{array}$ \\
\hline \multicolumn{7}{|l|}{ Costaceae (1/4\%) } \\
\hline $\begin{array}{l}\text { Costus spectabilis (Frenzl.) } \\
\text { K.Schum. }\end{array}$ & Savane & $\begin{array}{l}\text { Racine } \\
\text { tubérisée }\end{array}$ & $\mathrm{Hv}$ & GC & $\begin{array}{l}\text { Lubatabata, } \\
\text { Kimbatata }\end{array}$ & Bouenza \\
\hline \multicolumn{7}{|l|}{ Euphorbiaceae (3 / 12\%) } \\
\hline $\begin{array}{l}\text { Euphorbia cf. unispina } \\
\text { N.E.Br.* }\end{array}$ & Savane & Tige & $\mathrm{Hv}$ & $\mathrm{Cg}$ & Kihissa, Oleli & $\begin{array}{l}\text { Bouenza, } \\
\text { Cuvette- } \\
\text { Ouest, } \\
\text { Niari }\end{array}$ \\
\hline Euphorbia trigona Haw.* & Culture & Tige & ar & Aftr & $\begin{array}{l}\text { Kihisa, } \\
\text { Niondo, } \\
\text { Kikelo }\end{array}$ & $\begin{array}{l}\text { Bouenza, } \\
\text { Niari }\end{array}$ \\
\hline Manihot esculenta Crantz* & Culture & Feuille & ar & Pant & Ntôba & Pool \\
\hline \multicolumn{7}{|l|}{ Fabaceae (4 / 16\%) } \\
\hline Leptoderris sp.* & Forêt & $\begin{array}{l}\text { Tige } \\
\text { fraîche }\end{array}$ & $\mathrm{L}$ & - & $\begin{array}{l}\text { Mudunvu, } \\
\text { Mbaka ya } \\
\text { sangi }\end{array}$ & Pool \\
\hline $\begin{array}{l}\text { Pentaclethra macrophylla } \\
\text { Benth. }\end{array}$ & Forêt & Fruit & $\mathrm{Ar}$ & GC & $\begin{array}{l}\text { Mubala,Omba } \\
\text { la, Pandzou } \\
\text { katéka }\end{array}$ & Kouilou \\
\hline Tephrosia vogelii Hook.f. & Culture & Feuille & ar & Aftr & Bumi, Mbaka & Congo \\
\hline $\begin{array}{l}\text { Tetrapleura tetraptera (Schum. } \\
\text { \& Thonn.) Taubert }\end{array}$ & Forêt & Fruit & $\mathrm{Ar}$ & $\mathrm{Cg}$ & Yhaga, Yaga & Kouilou \\
\hline
\end{tabular}




\begin{tabular}{|c|c|c|c|c|c|c|}
\hline Gentianaceae (2 / 8\%) & & & & & & \\
\hline $\begin{array}{l}\text { Anthocleista schweinfurthii } \\
\text { Gilg }\end{array}$ & Forêt & Fruit & $\mathrm{Ar}$ & Aftr & $\begin{array}{l}\text { Lekoli, } \\
\text { Mupukupuku }\end{array}$ & Kouilou \\
\hline Anthocleista vogelii Planch & Forêt & Fruit & $\mathrm{Ar}$ & Aftr & $\begin{array}{l}\text { Lekoli, } \\
\text { Mupukupuku }\end{array}$ & Kouilou \\
\hline \multicolumn{7}{|l|}{ Loganiaceae (2 / 8\%) } \\
\hline Strychnos aculeata Solered & Savane & Fruit & $\mathrm{Li}$ & Gc & Inja, Ébômi & Kouilou \\
\hline $\begin{array}{l}\text { Strychnos camptoneura Gilg \& } \\
\text { Busse }\end{array}$ & Forêt & Fruit & $\mathrm{Li}$ & $\mathrm{Gc}$ & Inja, Ébômi & Kouilou \\
\hline \multicolumn{7}{|l|}{ Phyllanthaceae (2 / 8\%) } \\
\hline Bridelia ferruginea Benth. & Savane & Écorce & ar & Aftr & $\begin{array}{l}\text { Kihala, } \\
\text { Kikolokoto }\end{array}$ & $\begin{array}{l}\text { Bouenza, } \\
\text { Niari }\end{array}$ \\
\hline $\begin{array}{l}\text { Bridelia micrantha (Hochst.) } \\
\text { Baill. }\end{array}$ & Ripicole & Écorce & ar & & $\begin{array}{l}\text { Kikolokoto } \\
\text { kia hemboke }\end{array}$ & $\begin{array}{l}\text { Bouenza, } \\
\text { Niari }\end{array}$ \\
\hline \multicolumn{7}{|l|}{ Rubiaceae (1 / 4\%) } \\
\hline $\begin{array}{l}\text { Brenania brieyi (De Wild.) } \\
\text { E.Petit }\end{array}$ & Forêt & Fruit & $\mathrm{Ar}$ & $\mathrm{Gc}$ & $\begin{array}{l}\text { Molonjo, } \\
\text { Ombenda }\end{array}$ & $\begin{array}{l}\text { Kouilou } \\
\text { Sangha }\end{array}$ \\
\hline \multicolumn{7}{|l|}{ Rutaceae (2 / 8\%) } \\
\hline $\begin{array}{l}\text { Zanthoxylum gilletii (De Wild.) } \\
\text { Waterman }\end{array}$ & Forêt & Écorce & $\mathrm{Ar}$ & $\mathrm{Gc}$ & Bongo, Diaza & $\begin{array}{l}\text { Cuvette- } \\
\text { Ouest, } \\
\text { Sangha }\end{array}$ \\
\hline $\begin{array}{l}\text { Zanthoxylum heitzii (Aubr. \& } \\
\text { Pellegr.) Waterman }\end{array}$ & Forêt & Écorce & $\mathrm{Ar}$ & $\mathrm{Cg}$ & Bongo, Diaza & $\begin{array}{l}\text { Cuvette- } \\
\text { Ouest, } \\
\text { Sangha }\end{array}$ \\
\hline
\end{tabular}

(*) taxons à toxicité très aiguë et rémanent entraînant mort d'hommes

Tableau 2 : Plantes ichtyocides aux vertus inexplorées des communautés linguistiques enquêtées.

\begin{tabular}{|c|c|c|c|c|c|}
\hline Taxons & Habitat & $\begin{array}{l}\text { Organes } \\
\text { utilisés }\end{array}$ & T.M & T.P & $\begin{array}{l}\text { Nom } \\
\text { vernaculaire }\end{array}$ \\
\hline \multicolumn{6}{|l|}{ Cucurbitaceae (1) } \\
\hline Momordica charantia $\mathrm{L}$. & Savane & $\begin{array}{l}\text { Feuille \& } \\
\text { fruit sec }\end{array}$ & Hgr & $\mathrm{GC}$ & Dimbusimbusi \\
\hline \multicolumn{6}{|l|}{ Fabaceae (4) } \\
\hline Parkia filicoidea Welw. ex Oliv. & Forêt & Fruit & $\mathrm{Ar}$ & GC & - \\
\hline Indigofera hirsuta $\mathrm{L}$. & Savane & Fruit & S/ar & & - \\
\hline Pachyelasma tessmannii Harms & Forêt & Écorce & $\mathrm{Ar}$ & GC & Ediouk \\
\hline $\begin{array}{l}\text { Prosopis africana (G. \& Perr.) } \\
\text { Taub. }\end{array}$ & Forêt & Fruit & ar & GC & - \\
\hline \multicolumn{6}{|l|}{ Sapindaceae (1) } \\
\hline $\begin{array}{l}\text { Blighia welwitschii (Hiern) } \\
\text { Radlk. }\end{array}$ & Forêt & Fruit & $\mathrm{Ar}$ & Gc & Toko \\
\hline \multicolumn{6}{|l|}{ Rubiaceae (1) } \\
\hline $\begin{array}{l}\text { Sarcocephalus latifolius (Smith) } \\
\text { Bruce }\end{array}$ & Savane & Écorce & ar & Aftr & Mutumbi \\
\hline $\begin{array}{l}\text { Rutaceae (1) } \\
\text { Syzygium guineense (Willd.) DC }\end{array}$ & $\begin{array}{l}\text { Savane } \\
\& \text { forêt }\end{array}$ & Écorce & ar & $\mathrm{GC}$ & Mukisu \\
\hline
\end{tabular}


V. KIMPOUNI et al. / Int. J. Biol. Chem. Sci. 5(3): 979-990, 2011

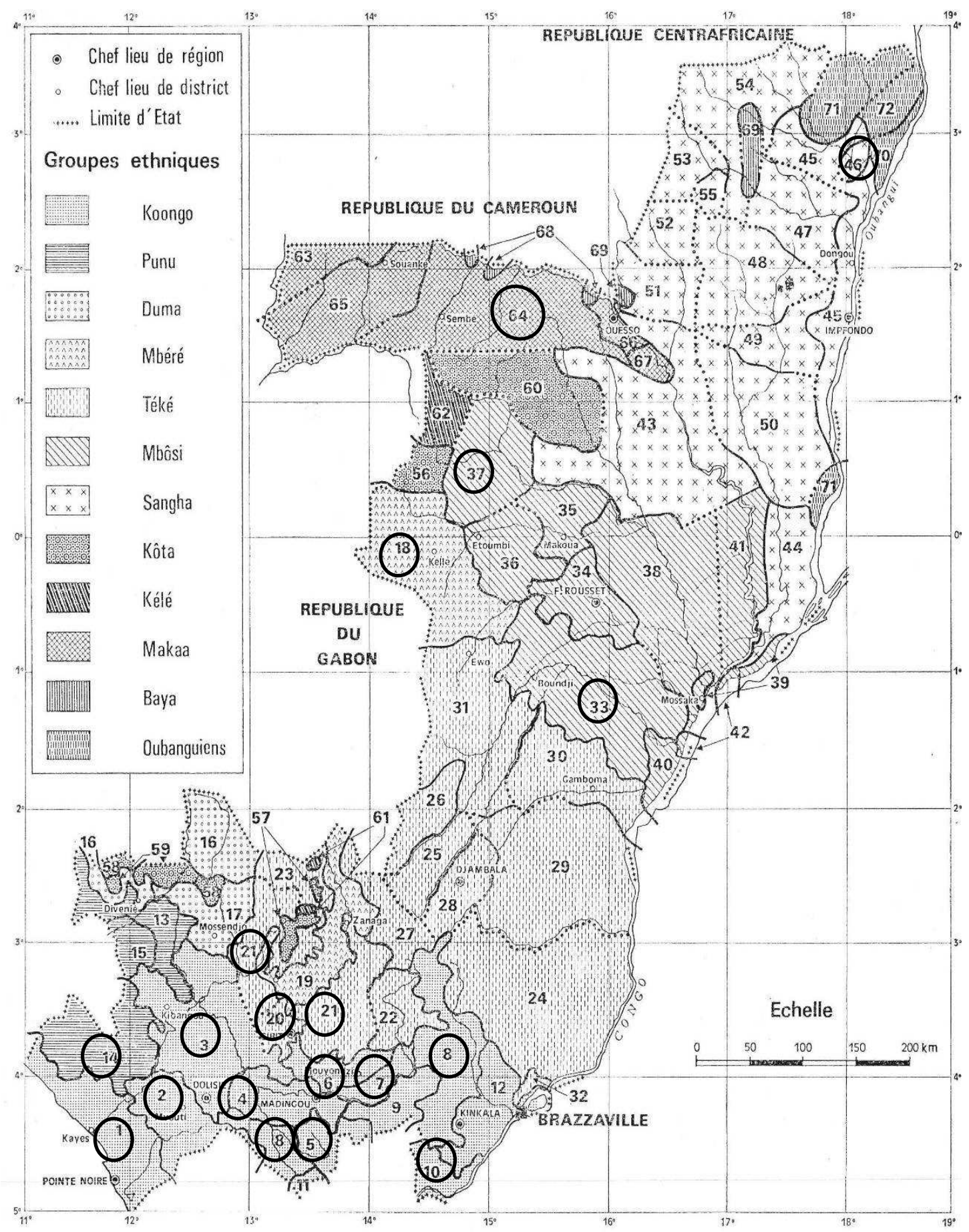

Figure 1 : Répartition ethnographique du Congo et communautés ethnico-linguistiques enquêtés (selon Bouquet, 1969). 


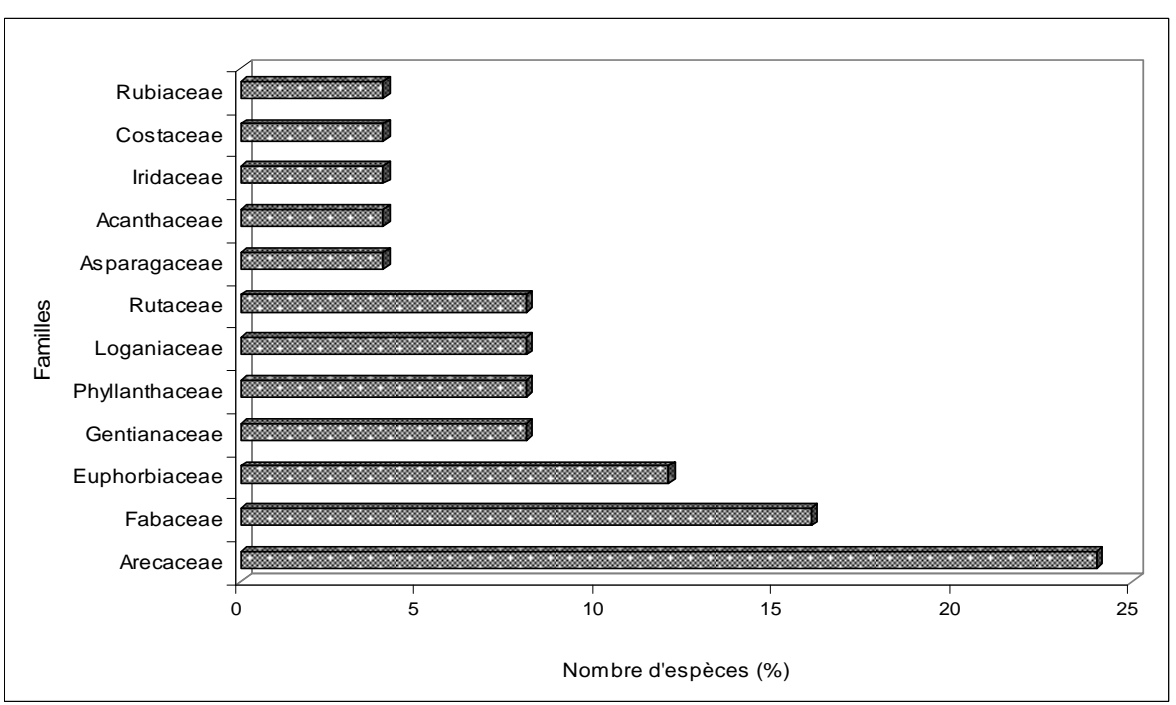

Figure 2 : Spectre de distribution de la diversité floristique.

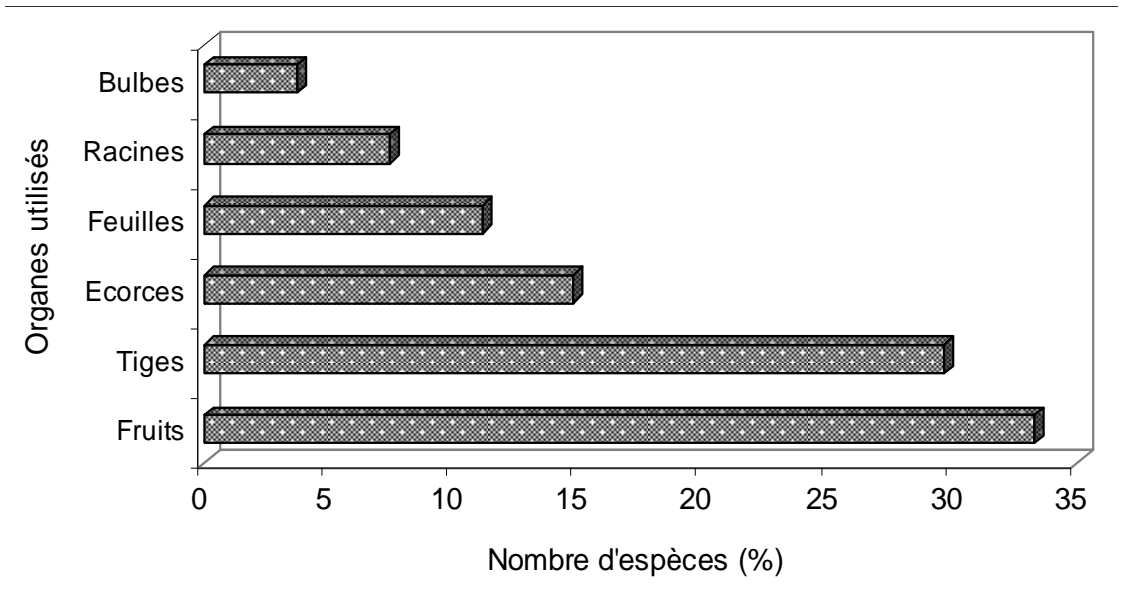

Figure 3 : Importance des organes utilisés.

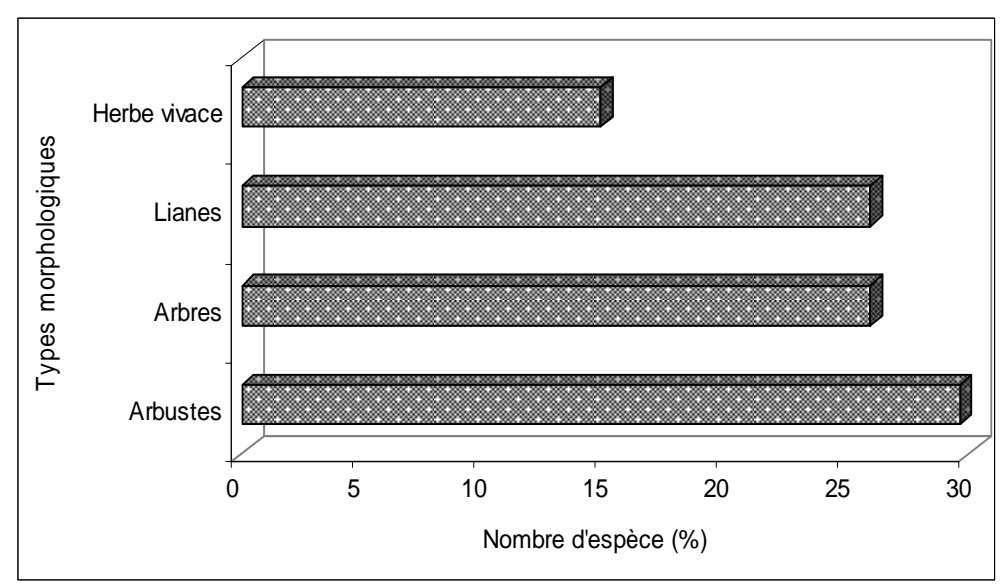

Figure 4 : Distribution des espèces en types morphologiques. 

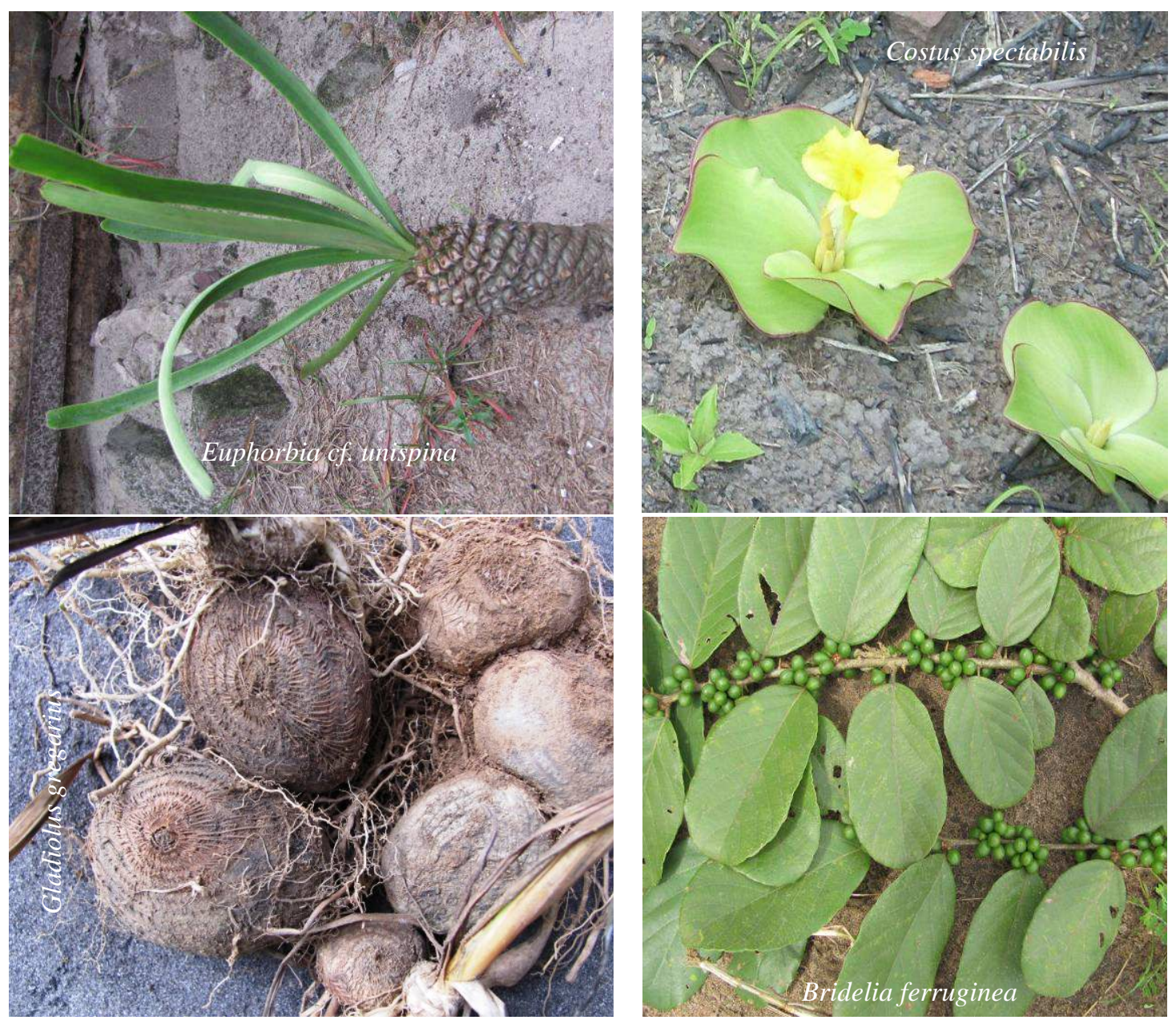

Planche 1: Vues de quatre espèces ichtyotoxiques utilisées par les communautés savanicoles (Photos V. Kimpouni)

\section{DISCUSSION \\ Biodiversité végétale et empirisme d'exploitation}

L'inventaire botanique révèle 26 espèces exploitées traditionnellement au Congo, pour leurs propriétés ichtyotoxiques, et dont chacune équivaut à une recette. L'analyse des données de la littérature montre que 12 de ces espèces, soit $46,15 \%$ sont citées pour la première fois au Congo, comme ichtyocides. Il s'agit de Bridelia sp., Calamus deeratus, Costus spectabilis, Eremospatha sp., Gladiolus gregarius, Leptoderris sp., Manihot esculenta, Urginea altissima et Raphia sp.. De ces nouvelles citations, les taxons se développant en savane ont une contribution de $41,67 \%$, alors que les $58,33 \%$ restant représentent les taxons forestiers, dont 50\% connus uniquement de la communauté ethnico-linguistique Koongo. En dépit du fait que ces plantes soient communes, aux différents écosystèmes forestiers et savanicoles congolais, les communautés riveraines n'ont pas toutes le même niveau de connaissance et d'exploitation de cette biodiversité. En effet, la communauté ethnicolinguistique Koongo avec $50 \%$ de ces nouvelles citations, est celle qui tire le plus des bénéfices. Elle est suivie par le groupe ethnique Mbôsi $(7,69 \%)$ et la communauté 
ethnico-linguistique Minkengue avec 3,85\%. Quant aux autres recettes, leurs usages caractérisent au moins deux groupes ethniques ou encore des communautés linguistiques d'un même groupe ethnique. Tel est le cas de: Bridelia sp., Gladiolus gregarius et Urginea altissima pour les communautés ethnicolinguistiques du groupe ethnique Koongo; Pentaclethra macrophylla, Tetrapleura tetraptera, Anthocleista sp. et Strychnos sp. pour deux communautés ethnico-linguistiques (Mvili et Yombé) du groupe ethnique Koongo ; Brenania brieyi pour les groupes ethniques Sangha et Mbére du nord Congo, et la communauté ethnico-linguistique Yombé (groupe ethnique Koongo) au sud-ouest; enfin, Euphorbia cf. unispina, une espèce de savane sur sol argileux, dont l'usage est commun au groupe ethnique Mbére du nord et les communautés ethnico-linguistiques du groupe Koongo au Sud, notamment les suundi, Doondo, Kougni et Kaamba.

Le rapport biodiversité végétale et empirisme d'exploitation révèle que les communautés ethnico-linguistiques voir les groupes ethniques du Congo, expriment des besoins identiques et recourent aux mêmes recettes pour satisfaire leurs exigences quotidiennes. Exploitant les produits naturels à portée de la main, ces communautés n'ont pas le même degré de prélèvement et de connaissances sur les atouts qu'offre la flore locale. Ces différences sont interprétées comme l'authentification de l'identité socioculturelle des communautés ethnicolinguistiques. Elles sont le synonyme des acquis naturels ou des savoirs endogènes des sociétés traditionnelles sur l'exploitation de la diversité floristique (Grenand et Prévost, 1994).

Une analyse des données écologiques et phytogéographiques des différents taxons montre qu'en dépit de quelques variantes spécifiques, les communautés forestières et savanicoles exploitent les mêmes espèces pour une même fin. Cependant, il est intéressant de souligner que des emprunts d'usages entre communautés ethnico-linguistiques d'une part, et, des groupes ethniques d'autre part, sont très plausibles. Ces échanges de connaissances sont matérialisés par la superposition des limites naturelles des territoires abritant ces espèces et le découpage ethnico-linguistique. C'est ainsi, qu'à défaut d'introduire Gladiolus gregarius ou Euphorbia cf. unispina dans leurs terroirs, la communauté Kaamba par exemple, se ravitaille sur les terres des communautés Doondo, Suundi et Kougni. Ces rapports peuvent être le symbole d'une acquisition nouvelle chez les Kaamba, découlant des contacts entre les membres des différentes communautés ethnico-linguistiques.

\section{Données ethnobotanique et socioculturelle}

La prospection ethnobotanique à travers la forêt et la savane congolaises relève des aspects d'usage de plantes presque désuètes. Ces pratiques très anciennes et connues de tous les peuples autochtones et locales sont séculaires sur tous les continents (Afrique, Amérique, Asie, Australie et Europe), comme le signalent Kerharo et al. (1960) et Stauch (1960). Elles représentent une valeur socioculturelle de ces communautés et mettent en exergue leurs connaissances ethnoanthropologiques, sur l'importance et l'exploitation de la biodiversité végétale. En dépit de cette valeur reconnue, ces notions sont en passe d'être oubliées car supplantées par les produits manufacturés, facteur limitant le transfert des acquis endogènes au sein des sociétés où l'oralité et l'empirisme sont le socle des savoirs. Dans les études ethnobotaniques, on relèvera que les plantes ichtyocides sont presque effacées par rapport aux aspects alimentaires, phytopharmaceutiques et artisanaux, qui font l'objet de plusieurs travaux (Bouquet, 1969 ; Bouquet et al., 1971; Bouquet et fournet, 1975 ; Adjanohoun et al., 1988; Kimpouni et Koubouana, 1997 ; Kimpouni, 1999) .

Liées aux trois savoirs (être, faire et vivre), ces connaissances qui sont un vrai capital de l'identité culturelle des communautés et/ou des groupes ethnicolinguistiques, subissent une forte érosion de valeur. Ce déclin est accéléré par le fait que 
l'usage de ces plantes ou mieux les parties de pêche impliquant ces organes, étaient liées à certaines cérémonies spécifiques aux us et coutumes qui n'ont plus cours de nos jours. C'est ainsi qu'est devenu désuet le caractère sacré, qui voilait l'utilisation de ces plantes et des produits de cette pêche. Suite à la désacralisation des vertus des différentes plantes, ces parties de pêche sont actuellement dévolues presque exclusivement aux femmes et aux enfants (Kerharo et al., 1960 ; Stauch, 1960). Outre les secrets y associés, plusieurs plantes à vertus ichtyocides ne sont pas révélées à quiconque, suite à leur forte toxicité pour l'homme. Ainsi, nous enregistrons pour la première fois des spécificités, du groupe ethnique/linguistique Koongo, relatives à Manihot esculenta, Eremospatha sp., Calamus deeratus et Leptoderris sp.

L'analyse de la distribution phytogéographique des taxons couplée aux données de la littérature, relève que plusieurs plantes ichtyotoxiques dont les vertus sont prouvées, ne sont pas en usage au sein de notre territoire d'étude, quoique présentes en quantité et en qualité (Tableau 2). Ces différences sont le signe caractéristique des valeurs intrinsèques propres aux communautés. En effet, ces connaissances ne relevant pas toujours du caractère populaire, leur exploitation fut souvent clanique et transmise suivant des rituels précis et hiérarchisés. C'est ainsi que plusieurs plantes présentes dans nos écosystèmes dont les vertus piscicides sont prouvées ailleurs ne sont pas connues des communautés disséminées à travers la forêt congolaise. Cette lecture des habitudes et différences observées à travers la forêt, est aussi vraie pour les communautés savanicoles.

Les travaux d'inventaire ethnobotanique sur la valorisation des savoirs endogènes des populations locales et autochtones, au Congo, se focalisent presque toujours dans les zones forestières. Ceci porte à croire que les savanes ne regorgent pas des plantes utiles et les populations qui y vivent n'ont aucune emprise sur la flore. Cette étude montre que les populations exploitent la diversité floristique environnante, quel que soit l'écosystème en présence. Excepté Euphorbia trigona et Tephrosia vogelii qui sont devenues cultivées, toutes les autres sont spontanées et leurs usages se limitent à l'aire d'influence des communautés. Nonobstant les variations que l'on enregistre entre les communautés, les espèces forestières, plus nombreuses, sont la panacée des populations des massifs forestiers (Hecketsweiler et Mokoko-Ikonga, 1991). Par contre, celles vivant en savanes se contentent en général de Bridelia sp., Costus spectabilis, Gladiolus gregarius, Euphorbia sp., Manihot esculenta, et Urginea altissima.

\section{Propriétés phytochimiques}

L'usage des plantes piscicides dans cette partie de pêche n'est pas sans conséquences sur la biodiversité biologique aquatique. La toxine n'étant pas sélective et spécifique à une catégorie donnée de la biodiversité, les effets sur la faune aquatique ne sont pas négligeables (Kerharo et al., 1960 ; Stauch, 1960). Les données de l'analyse chimique de plusieurs plantes ichtyotoxiques montrent différents produits dont les activités sont arthropicides (insecticides et antipsoriques), vermicides (anthelminthiques et plathelminthes) et antiseptiques (protozoaires). Les composés souvent mis en évidence sont la roténone et ses dérivés, très répandue chez les Fabaceae (Raponda et Sillans, 1961), les saponides (couramment présents dans plusieurs familles de plantes). Outre ces principaux composés, on relève les alcaloïdes (la strychnine, la brucine), les résines (Euphorbiaceae: Bridelia $s p$. et Euphorbia sp.), les acides et les tanins (Kerharo et al., 1960 ; Mabberley, 1993). Dans la plupart des recettes piscicides, la consommation des prises serait sans danger, excepté celles issues de l'usage de Euphorbia $s p$. . En effet, la toxicité du latex des euphorbes (Euphorbia sp.) et celle d'autres produits comme les feuilles de Tephrosia vogelii ou les écorces de Bridelia ferruginea utilisées comme ichtyocide est prouvée par toutes les communautés l'ayant expérimenté (Kerharo et al., 1960). Les empoisonnements par 
contamination via la consommation du poisson étant courants; celui-ci doit être vidé sans délais dès le ramassage, avant que mort s'ensuive. Sans cette précaution, le poisson est impropre à la consommation. Ainsi, en tenant compte de la rémanence du produit et des caractéristiques biologiques des espèces de poissons, une partie non négligeable de cette pêche est toujours perdue. Consciente de la toxicité très aiguë et le pouvoir rémanent de certaines plantes utilisées, les communautés et/ou les groupes ethnico-linguistiques, se sont dotés d'un «code» de bonne conduite qui réglemente et/ou prohibe leur usage. Cet élément de gestion est relevé dans toutes les communautés pratiquant la pêche à base des plantes ichtyotoxiques.

En tenant compte des facteurs exogènes (pédo-climatiques) et intrinsèques liés à la diversité génétique, la composition phytochimique des individus d'une même espèce peut varier en quantité et en qualité (Bouquet et al., 1971 ; Bouquet et Fournet, 1975 ; Sofowora, 1996). Dans ce contexte, plusieurs espèces (Tableau 2) aux vertus piscicides prouvées (Kerharo et al., 1960 ; Stauch, 1960; Tailfer, 1989) ne sont pas exploitées dans notre région. Ces spécificités qui ne relèvent nullement de la méconnaissance (manque d'informations) uniquement, peuvent avoir un fondement écologique à défaut du savoir empirique. En effet, nous avons noté l'appropriation de certains usages, notamment en milieu forestier, suite aux déplacements et aux contacts des populations.

\section{Conclusion}

Aspect moins évoqué au cours des études sur les savoirs endogènes des communautés forestières et savanicoles sur l'exploitation de la diversité floristique, cette étude s'inscrit dans deux directions convergentes. Il s'agit de l'amélioration des connaissances sur l'usage de la biodiversité végétale du Congo, d'une part, et, d'autre part, la pérennisation des savoirs des peuples autochtones et locaux dont le caractère désuet ne fait aucun doute. Cette étude montre que toutes les populations forestières, du nord au sud, exploitent pour une même fin, les mêmes espèces à quelques exceptions près. Aussi, elle met un accent particulier sur les savoirs des populations de savanes sur les ichtyocides végétales. En rapport avec l'aire de distribution des différentes plantes, notamment les forestières, il en découle que leur vertu piscicide n'est pas méconnue des communautés humaines. Quant aux espèces savanicoles, leur citation comme ichtyotoxiques est le fondement de cette étude.

\section{REFERENCES}

Adjanohoun EJ, Ahyi AMR, Ake Asi L, Baniakina J, Chibon P, Cusset G, Doulou V, Enzanza A, Eyme J, Goudote E, Keita E, Mbemba C, Mollet J, Moutsambote JM, Mpati J, Sita P. 1988. Contribution aux Etudes Ethnobotaniques et Floristiques en République Populaire du Congo: Médecine Traditionnelle et Pharmacopée. ACCT: Paris.

APG III. 2009. An update of the Angiosperm phylogeny group classification for the orders and families of flowering plants: APG III. Botanical Journal of the Linnean Society, 161: 105-121.

Bouquet A, Fournet A. 1975. Recherches récentes sur les plantes médicinales congolaises. Fitoterapia, XLVI(6): 242246.

Bouquet A. 1969. Féticheurs et Médecine Traditionnelle du Congo (Brazzaville). Mém. ORSTOM 36: Paris.

Bouquet A, Cavé A, Paris R. 1971. Plantes médicinales du Congo Brazzaville (III). Plantes Médicinales et Phytothérapie, 4(2): 154-158.

Hecketsweiler P, Mokoko-Ikonga J. 1991. La Réserve de Conkouati: Congo, le Secteur Sud-Est. UICN: Gland, Suisse.

Grenand P, Prévost M-F. 1994. Les plantes colorantes utilisées en Guyane française. J. Agric. Trad. Bota. Appl., 36(1): 139172.

Kerharo J, Guichard F, Bouquet A. 1960. Les végétaux ichtyotoxiques (poisons de 
pêche). In Biologie Végétale et Matière Médicale, Kerharo J (éd). Bulletins et mémoires de l'École Nationale de Médecine et de Pharmacie de Dakar: Dakar; 313-329.

Kimpouni V, Koubouana F. 1997. Étude Ethnobotanique sur les Plantes Médicinales et Alimentaires dans et autour de la Réserve de Conkouati. PROGECAP/GEF-Congo, UICN.

Kimpouni V, Nguembo J. (sous presse). Aspects d'exploitation et d'utilisation artisanales des produits forestiers d'origine végétale à Lossi, Congo (Brazzaville). Annales UMNG (sous presse).

Kimpouni V. 1999. A preliminary market survey of non-wood forest products traded in the Pointe-Noire markets (Congo-Brazzaville). In Non-wood Forest Products of Central Africa. Current Research Issues and Prospects for Conservation and Development, Sunderland TCH, Clark LE, Vantomme P (eds). CARPE-FAO: Rome; $221-226$.
Mabberley DJ. 1993. The Plant-book: A Portable Dictionary of the Higher Plants. Cambridge University Press: London.

Martin GJ. 1995. Ethnobotany: A Manuel Methods. Chapman \& Hall: Lodon.

Raponda Walker A, Sillans R. 1961. Les Plantes Utiles du Gabon. Ed. Lechevalier: Paris.

Sofowora A. 1996. Plantes Médicinales et Médecine Traditionnelle d'Afrique. Karthala: Paris.

Stauch A. 1960. Les ichtyotoxiques dans la pêche africaine. 4è Colloque sur hydrobiologie et pêches en eau douce, Fort Lamy. CCTA/CSA, Paris.

Tailfer Y. 1989. La Forêt dense d'Afrique centrale: Identification Pratique des Principaux Arbres. CTA/ACCT, Paris.

UICN. 1996. L'Atlas pour la Conservation des Forêts Tropicales d'Afrique. UICN: Gland, Suisse.

Vennetier P. 1977. Atlas de la République Populaire $d u$ Congo. Éditions Jeune Afrique: Paris. 